\title{
Mirabegron: A Novel and Promising Medical Expulsive Treatment for Ureteral Stones?
}

\author{
Ahmet Urkmez1, Emre Tokuc1, Ramazan Topaktas1, Aytac Sahin² and Ozgur H. Yuksel3
}

\begin{abstract}
Medical expulsive therapy (MET) is used especially in distal ureteral stones to reduce colics and decrease the number of endourological surgical interventions. A broad spectrum of agents can be used for the relaxation and the dilatation of the ureter, reducing the intraureteric pressure. Alfa-blockers, calcium channel blockers, phosphodiesterase (PDE) inhibitors, and spasmolytics have been shown as effective in clinical trials on urolithiasis. It is a fact that the urothelium itself, the interstitial cells and the ureteric smooth muscle, have B-beta-2 and beta-3 adrenoreceptors. Stimulation of these receptors results in relaxation of the ureter. A recent beta-3 agonist, mirabegron, is commonly used for overactive bladder nowadays. The mechanism of action is adrenergic agonism that affects with the storage phase of the bladder, without interfering the voiding phase, which is regulated by parasympathetic pathways, commonly muscarinic. Agonism of the beta-3 receptors in the ureter has been shown to decrease the intraluminal pressure. By this mechanism, mirabegron can be thought as an alternative MET agent. Acting through different pathways, and having low adverse effect profile, can be thought as the most striking advantages of mirabegron as a MET. In vitro and in vivo trials should be conducted to support this hypothesis.
\end{abstract}

Key Words: Medical expulsive treatment, Mirabegron, Ureteral stone, Beta-3 adrenoreceptor.

\section{INTRODUCTION}

Medical expulsive therapy (MET), administering the drugs to help expel the stones, is being used widely throughout the world. Using alpha-blockers, calcium channel blockers, phosphodiesterase (PDE) inhibitors, and spasmolytics have been shown effective in clinical trials on urolithiasis. ${ }^{1} \mathrm{MET}$ intends to relax the ureteric smooth muscle and facilitates the ureteric stone to pass, decreasing the number of surgical interventions, and reducing the healthcare costs. Also MET helps shockwave lithotripsy (SWL) and the discomfort of the ureteral stents, improving the success rate and providing a better and faster outcome. 2,3

The ureter, delivering the urine from kidneys to the bladder, is a tube-shaped structure with an autonomous stimulated muscle layer and functions continuously with peristalsis by the effect of the pacemaker cells (Cajal) near ureteropelvic junction. The stimulus tends to spread with dual mesh-like neuronal networks throughout the ureter. Due to this complex neuronal anatomy, ureter contains many receptors; and neurotransmitters involved in the smooth muscle contraction and relaxation. Alpha- 1 adrenergic receptors, muscarinic

1 Department of Urology, Haydarpasa Numune Research and Training Hospital, Istanbul, Turkey

2 Department of Urology, Fatih Sultan Mehmet Research and Training Hospital, Istanbul, Turkey

${ }^{3}$ Department of Urology, Bahcesehir University, VM Medical Park Hospital, Istanbul, Turkey

Correspondence: Dr. Ahmet Urkmez, Department of Urology, Haydarpasa Numune Research and Training Hospital,

Kadikoy, Tr-34668 Istanbul, Turkey

E-mail:ahmeturkmez@hotmail.com

Received: April 24, 2018; Accepted: September 14, 2018 receptors, purinergic receptors, and histaminergic $(\mathrm{H} 1)$ receptors with serotonin, prostaglandin $\mathrm{F} 2 \mathrm{a}$, substance $P$, neurokinin $A$, neuropeptide $Y$ and rho-kinase pathway are involved in the contraction of the ureter and in contrast, beta- 2 / 3 receptors, histaminergic $(\mathrm{H} 2-\mathrm{H} 3)$ receptors with nitric oxide, prostaglandin (E1-E2), calcitonin gene-related peptide (CGRP), adenosine and vasociture inertial peptide (VIP) take part in relaxation. ${ }^{4}$ Passage through the ureter can be facilitated by blocking the contracting pathways or stimulating the relaxation cascade.

Alpha-blockers are widely used in lower urinary tract symptoms (LUTS) for benign prostate hyperplasia $(\mathrm{BPH})$. On the other hand, blocking alpha-adrenergic receptors in ureter helps the intraureteral pressure to decrease and the fluid passage per unit of time to increase. Nowadays, alpha-blockers in MET are efficient and preferable in clinical practice. Nifedipine is a calcium canal blocker used for the same purpose. Comparatively, it has limited effect on ureter smooth muscle compared with alpha-blockers. ${ }^{5}$ For this reason, the use of calcium channel blockers is now rare. Lately, PDE-5 inhibitors, like tadalafil, may seem to be beneficial in terms of MET.1,5 Wide spectrum of these drugs can lead the practitioners to prescribe combined therapy for stone passing, too.

The hypothesis: Beta- 3 receptors are commonly found in the detrusor, participating in bladder relaxation. 6 It has been shown that beta- 2 and beta-3 adrenoreceptor subtypes are detected in the ureteral smooth muscle, mediating the adrenergic stimulation causing ureteral relaxation. ${ }^{7}$ Moreover, urothelium and the interstitial cells themselves express beta- 3 adrenoreceptors more than the ureteral smooth muscle. This indicates that beta3 adrenoreceptors take part in the dynamics of the 
ureter. Beta- 3 receptors can also be found in ventriculi, human vasculature, brain, retinal endothelial cells, gastrointestinal tract, skeletal muscle and in brown and white adipose tissues. When compared, adipose tissues and urinary tract have higher density of the beta-3 receptors than other systems. 6,7

A selective beta- 3 agonist, mirabegron, is a trending drug, which is recently used in overactive bladder. Mirabegron was discovered in 2007, tends to have an agonist effect on beta-3 receptors and affects on storage phase of bladder and increases the bladder capacity without influencing the voiding phase and the bladder contractility. In contrast, antimuscarinics blocks the M3 cascade which contract detrusor. This effect, on the storage phase, is dose-dependent and reduces the micro-motion in the detrusor. 8

The relaxant effect of beta- 3 agonists in the ureter has been shown in several in vivo studies in dogs and pigs, where the intraluminal pressure in the ureteral has significantly decreased. 9 As mirabegron relaxes the ureteral muscles and dilates the ureter lumen by stimulating beta- 3 adrenoreceptors, it may serve as an effective and safe alternative for MET, which act with a totally different pathways.

\section{DISCUSSION}

A large proportion of anticholingergics, used for overactive bladder, have different adverse effects like dry-mouth, slowed gastrointestinal motility, blurred vision, and increased heart rate. Reviews and meta-analyses indicate that the the therapeutic dose of mirabegron, 50 $\mathrm{mg}$, is not associated with blood pressure changes, QT time or heart rate. ${ }^{10}$ However, there was a significant increase in heart rate when $100 \mathrm{mg}$ and $200 \mathrm{mg}$ of mirabegron was administered. This was not associated with increased risk of cardiovascular adverse effects. On the other hand, comparative studies of anticholinergics with mirabegron found that the risk of dry-mouth and constipation is much less with mirabegron. Moreover, being a beta- 3 agonist, mirabegron affects detrusor with a totally different pathway without manipulating the voiding phase. ${ }^{8}$

Classical METs contain alpha-blockers nowadays. Alpha-blockers have their own adverse effects due to their mechanism of action. Patients using these as MET may have retrograde ejaculation, nausea, dizziness and orthostatic hypotension. From this point of view, mirabegron seems to be safer to use in terms of sexual and cardiovascular adverse effects.

Matsumoto et al. classified ureters from nephrectomy and cystectomy specimens as proximal / middle and distal ureter, in their study. ${ }^{7}$ These ureters were exposed selective beta-2 and beta- 3 agonists in appropriate solutions to measure the contractions of ureters; and both beta-2 and beta-3 adrenoceptor agonists significantly decreased the contraction amplitudes. In addition, immunohistochemical studies on these ureters have shown that beta-3 adrenoceptors are found not only in ureter smooth muscle but also in the urothelium. Although there are studies that emphasise that there are beta-2 adrenoreceptors in the urothelium and smooth muscle, the high incidence of adverse effects such as nervousness, headache, tachycardia and palpitations make the clinicians hesitate to prescribe them as a MET. Despite activating the beta- 3 receptors at the ureter, relaxes the ureteric smooth muscle and regulates the ureteral peristalsis, there is no research or clinical trials done in terms of its use as MET. Nowadays, mirabegron lacks an indication as MET agent for stone expulsion resulting in few clinical trials in terms of ethical and legal issues. More in vitro and in vivo studies are needed on this subject.

In conclusion, as in overactive bladder, beta-3 adrenoreceptor agonists seem to be promising in MET, too. In addition, lower urinary tract symptoms, especially those associated with distal ureteral stones with significantly reduced quality of life, will respond to mirabegron use and improve the quality of life of the patient during follow-up.

\section{REFERENCES}

1. Türk C, Knoll T, Seitz C, Skolarikos A, Chapple C, McClinton S, et al. Medical expulsive therapy for ureterolithiasis: The EAU recommendations in 2016. Eur Urol 2017; 71:504-7.

2. Kroczak T, Scotland KB, Chew B, Pace KT. Shockwave lithotripsy: tech-niques for improving outcomes. World $J$ Urol 2017; 35:1341-6.

3. Lim KT, Kim YT, Lee TY, Park SY. Effects of tamsulosin, solifenacin, and combination therapy for the treatment of ureteral stent related discomforts. Korean J Urol 2011; 52:485-8.

4. Canda AE, Turna B, Cinar GM, Nazli O. Physiology and pharmacology of the human ureter: Basis for current and future treatments. Urol Int 2007; 78:289-98.

5. Wang H, Man LB, Huang GL, Li GZ, Wang JW. Comparative efficacy of tamsulosin versus nifedipine for distal ureteral calculi: A meta-analysis. Drug Des Devel Ther 2016; 10:1257-65.

6. Coelho A, Antunes-Lopes T, Gillespie J, Cvuz F. Beta-3 adrenergic receptor is expressed in acetylcholine-containing nerve fibers of the human urinary bladder: An immunohistochemical study. Neurourol Urodyn 2017; 36:1972-80.

7. Matsumoto R, Otsuka A, Suzuki T, Shinbo H, Nizuno T, Kurita $Y$, et al. Expression and functional role of $\beta 3$-adrenoceptors in the human ureter. Int J Urol 2013; 20:1007-14.

8. Sebastianelli A, Russo GI, Kaplan SA, Mc Vary KT, Moncada I, Gravas $S$, et al. Systematic review and meta-analysis on the efficacy and tolerability of mirabegron for the treatment of storage lower urinary tract symptoms/ overactive bladder: Comparison with placebo and tolterodine. Int J Urol 2018; 25: 196-205

9. Wanajo I, Tomiyama Y, Yamazaki Y, Kojima M. Ureteral selectivity of intravenous beta-adrenoceptor agonists in pig model of acute ureteral obstruction: comparison of KUL-7211, a selective beta2/beta3 agonist, with isoproterenol, terbutaline, and CL-316243. Urology 2011; 77:e1-6.

10. Michel MC, Gravas S. Safety and tolerability of beta3adrenoceptor agonists in the treatment of overactive bladder syndrome - insight from transcriptosome and experimental studies. Expert Opin Drug Saf 2016; 15:647-57.

$$
\text { .......... }
$$

